Revista de Negócios_ISSN 1980.4431_vol. 19, n. 4, p. 43_63 2014_DOI:10.7867/1980-

4431.2014v19n4p43_63

\title{
O setor brasileiro de nanotecnologia: Oportunidades e desafios
}

\section{The Brazilian nanotechnology industry: Opportunities and challenges}

\author{
Marcos Roberto Piscopo \\ UNINOVE Universidade Nove de Julho - Brasil \\ piscopomr@yahoo.com \\ Cláudia Terezinha Kniess \\ UNINOVE Universidade Nove de Julho - Brasil \\ kniesscl@yahoo.com.br \\ César Augusto Biancolino \\ UNINOVE Universidade Nove de Julho - Brasil \\ biancolino@gmail.com \\ Claudia Echevengua Teixeira \\ UNINOVE Universidade Nove de Julho - Brasil \\ clateixeira@gmail.com
}

Recebido em 8 de março de 2014. Alterado em 2 de outubro de 2014. Aprovado em 11 de novembro de 2014. Editores Responsáveis: Edson Roberto Scharf, Dr. e Marianne Hoeltgebaum, Dr.

Processo de avaliação por double blind review

\section{Resumo}

Este trabalho visa avaliar estrategicamente o setor brasileiro de nanotecnologia, para identificar oportunidades e desafios. A partir de uma pesquisa documental, de natureza exploratória, analisou-se o setor de nanotecnologia, mais especificamente, sua estrutura, sua atratividade e potenciais estratégias para alteração de sua estrutura. Constatou-se, o Brasil ainda necessita se posicionar mais claramente quanto aos seus objetivos e suas políticas de apoio a esse setor para capitalizar oportunidades e minimizar riscos associados à aplicação da nanotecnologia, incluindo mas não se limitando (i) ao estabelecimento de marcos regulatórios que definam claramente as possibilidades de sua aplicação e as responsabilidades sobre os riscos assumidos, (ii) à definição de políticas de investimento que viabilizem o avanço científico e a difusão tecnológica da nanotecnologia e (iii) à criação de mecanismos de governança que facilitem a comunicação com a sociedade e proporcionem maior transparência sobre os benefícios e riscos decorrentes da aplicação da nanotecnologia.

Palavras-chave: setor, nanotecnologia, oportunidades, desafios

\section{Abstract}

The present work aims at strategically assessing the Brazilian nanotechnology industry with the purpose of identifying the main opportunities and challenges. Through a documental 
research, of exploratory nature, we analyzed the nanotechnology industry, more specifically its structure, attractiveness and potential strategies to alter industry structure. We found that Brazil needs a transparent strategic positioning to clarify its goals and support policies for the nanotechnology industry in order to help it to capitalize opportunities and minimize risks related to the application of nanotechnology including but not limited to the (i) establishment of regulatory milestones that define possible applications and responsibilities for the risks taken, (ii) definition of investment policies that make feasible the nanotechnology scientific advance and technological diffusion, and (iii) creation of governance mechanisms that help the communication with the society and enable greater transparency with regard to the benefits and risks deriving from the nanotechnology application.

Keywords: industry, nanotechnology, opportunities, challenges

\section{Introdução}

A Nanociência e Nanotecnologia se refere ao estudo e as aplicações de materiais e dispositivos com dimensões físicas de até 100 nanômetros. As nanopartículas possuem propriedades mecânicas, óticas e químicas específicas e de grande aplicabilidade para os setores químico, têxtil, automotivo, citando apenas alguns exemplos. Alguns produtos finais que resultam da nanotecnologia incluem tecidos que não molham, polímeros resistentes tanto quanto o aço e o alumínio, películas para vidros e cosméticos cujas partículas penetram nos poros da pele, entre outros. Existem previsões que o mercado de nanotecnologia alcancará 2,9 trilhões de dólares e o Brasil não parece estar preparado para isso visto que não possui uma agenda apropriada quando comparada com os países desenvolvidos. Portanto, este trabalho busca entender a situação atual do setor de nanotecnologia no Brasil e o que se faz necessário para capitalizar as oportunidades.

A literatura sobre nanotecnologia ainda se encontra em crescimento, com destaque para os pontos positivos e nega- tivos com relação à sua aplicação. Por um lado, vários autores defendem sua aplicação à agricultura (Rochman et al, 2011; Wilson, 2012), aos alimentos (Kuzma; Romanchek; Kokotovich, 2008; Sozer; Kokini, 2010; Stampfli; Siegrist; Kastenholz, 2010), às embalagens para alimentos (Buzby, 2010; Food Engineering \& Ingredients, 2011; Stampfli; Siegrist; Kastenholz, 2010), ao cimento e concreto (Erdogan, 2011), aos eletrônicos (Doering, 2011), às tendências e o futuro da nanotecnologia (Lindquist; Mosher-Howe; Liu, 2010; Meyer; Libaers; Park, 2011; Priest et al, 2011; Thomas; Acuña-Narvaes, 2006; Van Lente, 2006; Zawislak et al, 2010), à inovação (Khadzhiev, 2011; Moinddin; Rashdi; Bhutto, 2010; Woolley; Rottner, 2008), à comercialização (Rasmussen, 2007; Uranga; Kerexeta; CampàsVelasco, 2007), às políticas de desenvolvimento (Ghazinoory; Ghazinouri, 2009; Ghazinoory; Soofi, 2012; Sá; Geiger; Hallacher, 2008; Stinnett, 2012; Wang; Shapira, 2012), entre outros tópicos relevantes.

Por outro lado ainda existem vários questionamentos sobre à aplicabilidade da nanotecnologia, especialmente com respeito ao consumo de produtos à base de nanotecnologia (Batitsta; Peppe, 2014; Foladori; Figueroa; Edgard; Invernizzi, 2014; Reisch; Scholl; Bietz, 2011; Throne-Holst; Strandbakken, 2009; Yawson; Kuzma, 2010), ao emprego de nanomateriais (Handy; Shaw, 2007; Shatkin; North, 2010; van Broekhuizen; Reijnders, 2011), ao registro de patentes (Featherstone, 2004; Fernández-Ribas, 2010; Igami; Okazaki, 2007; Preschitscheck; Bresser, 2010; Schellekens, 2010), aos riscos (Pyrrho; Schramm, 2012; Kuzma; Priest, 2010; Tsai, 2012; Williams et al, 2010; Santos, 2010), aos riscos associados à comunicação e opinião pública (Conti; Satterfield; Harthorn, 2011; Doubleday, 2007; Ott; Papilloud, 2008; Pidgeon; RogersHayden, 2007; Pidgeon; Harthorn; 
Satterfield, 2011, Xenos et al, 2011), à difusão do conhecimento (Liu et al, 2011), aos investimentos (Festel; Kratzer, 2012; Munari; Toschi, 2010), aos aspectos éticos (Fink; Harms; Hatak, 2012; Linton; Walsh, 2012; Lu et al, 2012), à responsabilidade social corporativa (Groves et al, 2011), à segurança do paciente (Altenstetter, 2011; Nerlich; Clarke; Ulph, 2007), aspectos regulatórios (Falkner; Jaspers, 2012; Sylvester; Abbott; Marchant, 2009), apenas para citar alguns temas de importância.

Diante das amplas possibilidades para aplicação da nanotecnologia e das incertezas associadas a elas, verifica-se uma lacuna de conhecimento com relação ao ambiente brasileiro de negócios desse setor. Portanto, este trabalho busca avaliar estrategicamente o setor brasileiro de nanotecnologia para melhor compreender suas oportunidades e desafios.

Além desta seção que contextualiza o tema nanotecnologia, demonstra o estado da arte da literatura e apresenta o objetivo do estudo, este trabalho contém mais quatro seções. A segunda aborda uma revisão teórica sobre análise estratégica e nanotecnologia. Na terceira, demonstra-se os procedimentos metodológicos empregados. A quarta seção acomoda a apresentação e análise dos resultados. Finalmente, na quinta seção, faz-se as considerações finais, ressaltando as conclusões, contribuições, limitações e possibilidades de novas pesquisas.

\section{Revisão Teórica}

Este tópico cobre os temas análise estratégica e nanociência e nanotecnologia. $\mathrm{Na}$ parte de análise estratégica aborda-se o ambiente externo e o ambiente setorial e, na parte de nanociência e nanotecnologia, cobre-se seus aspectos essenciais e seu papel para a sociedade.

\subsection{Análise Estratégica}

A análise estratégica é uma etapa importante da administração estratégica, juntamente com as decisões e ações que uma organização toma em busca da criação e sustentação de vantagens competitivas (Dess; Lumpkin; Eisner, 2007). A análise estratégica é um passo relevante para passar do diagnóstico para a consideração de novas ideias e proposições estratégicas, visto que as últimas envolvem ideias discretas e planos de ação que impactarão os componentes da estratégia e, consequentemente, a estratégia do negócio todo (Fry; Killing, 2000). O objetivo da análise estratégica é fornecer subsídios para que a empresa possa atuar de forma eficaz perante ameaças ambientais e capitalizar oportunidades que proporcionadas pelo ambiente (Wright; Kroll; Parnell, 2000).

A análise ambiental envolve os meios pelos quais os gestores que atuam nos setores privado e público buscam entendimento sobre as incertezas do ambiente de negócios no qual suas organizações se inserem, o que apresenta certa dificuldade porque existe grande diversidade de influências sobre o ambiente e, em muitos casos, estas estão interconectadas; portanto, a compreensão dessas conexões é essencial para a construção de um quadro estratégico do ambiente empresarial (Johnson; Scholes; Whittington, 2007).

A finalidade da análise ambiental é auxiliar as organizações a serem eficientes e eficazes no alcance de suas metas, sendo que os tipos de análises dependem da qualidade das atividades desempenhadas e, portanto, o processo de análise ambiental deve ser avaliado assim como as demais atividades organizacionais (Certo et al, 2010).

\subsubsection{O Ambiente Externo}

O ambiente geral contempla aspectos externos à organização que apre- 
sentam escopo amplo e sobre os quais a organização não possui controle (Certo et al, 2010). O ambiente geral aborda as tendências amplas no contexto em que a empresa se insere e que podem impactar suas escolhas estratégicas, portanto a análise de oportunidades e ameaças deve ocorrer a partir da compreensão do ambiente geral (Barney; Hesterly, 2007). O entendimento do ambiente externo é crucial para a formulação de estratégias eficazes visto que esse é a fonte de oportunidades e ameaças para a empresa e, portanto, pode comprometer o sucesso da implementação da estratégia e influenciar a lucratividade da empresa (Carpenter; Sanders, 2007).

A importância da análise macroambiental do setor não está somente em identificar as condições atuais mas em tentar prever a ocorrência de mudanças e tendências futuras (Wright; Kroll; Parnell, 2000). A partir da análise externa a empresa pode identificar as ameaças e oportunidades mais relevantes para seu ambiente competitivo, avaliar a evolução deste ambiente e suas implicações para as referidas ameaças e oportunidades (Barney; Hesterly, 2007). "As empresas bem-sucedidas coletam as informações necessárias para entender cada segmento e suas implicações para a seleção e implantação das estratégias adequadas" (Hitt; Ireland; Hoskisson, 2008, p. 35).

"Uma oportunidade é uma condição no ambiente geral, que se explorada, ajuda a empresa a obter competitividade estratégica" (Hitt; Ireland; Hoskisson, 2008, p. 37). "Uma ameaça é uma condição do ambiente geral que pode impedir os esforços de uma empresa em obter competitividade estratégica" (Hitt; Ireland; Hoskisson, 2008, p. 37). Uma ameaça ambiental pode surgir a partir de indivíduos, grupos ou organizações externas à empresa que visam reduzir seu desempenho, pois forçam a elevação dos custos, a redução das receitas ou outras formas de redução do desempenho da empresa tendendo a aumentar a competitividade setorial e pressionar o desempenho da empresa para uma zona de paridade competitiva (Barney; Hesterly, 2007).

Existem diversas fontes para análise do ambiente geral, como consulta a materiais impressos (jornais, pesquisas acadêmicas, publicações comerciais e de opinião pública), visitas a feiras comerciais, fornecedores, clientes e funcionários de empresas (Hitt; Ireland; Hoskisson, 2008).

\subsubsection{O Ambiente Setorial}

Um setor industrial é "um grupo de empresas que produzem mercadorias ou oferecem serviços concorrentes" (Wright; Kroll; Parnell, 2000, p. 47), ou seja, "uma indústria (setor) é um grupo de empresas que fabrica produtos que são substitutos próximos" (Hitt; Ireland; Hoskisson, 2008, p. 47).

As características fundamentais de um setor envolvem os fatores que apresentam relevância para o desempenho da empresa em dada situação, ou seja, as características distintivas que aparecem em uma fotografia do setor (Carpenter; Sanders, 2007). Com o avanço da concorrência as empresas de setor se influenciam, visto que elas adotam várias estratégias competitivas para obter competitividade estratégica e retornos acima da média (Hitt; Ireland; Hoskisson, 2008). Considerando que uma empresa pode competir em vários mercados ao mesmo tempo deve-se analisar cada mercado isoladamente visto que os competidores e a natureza da competição altera de mercado para mercado (Besanko et al, 2006).

Algumas abordagens para análise setorial incluem o modelo estruturaconduta-desempenho (Bain, 1968), o modelo das forças que dirigem a concorrência (Porter, 1980), a hipercompetição (D’Aveni, 1995), a coopetição (Brandenburger; Nalebuff, 1996), o 
cenário dos negócios (Ghemawat, 2007), a evolução dos setores (McGahan, 2004), entre outros. Contudo, "as análises eficazes da indústria são resultado de estudos e interpretações meticulosas dos dados e informações de várias fontes" (Hitt; Ireland; Hoskisson, 2008, p. 56).

Joe Bain, economista de Harvard, por meio de um estudo empírico com um número limitado de variáveis estruturais buscou entender as relações entre a estrutura e o desempenho de um setor (Ghemawat, 2007). O modelo estruturaconduta-desempenho (E-C-D) envolve a estrutura (estrutura do setor, mensurada por meio do número de concorrentes, a heterogeneidade dos produtos e os custos de entrada e de saída), a conduta (estratégias adotadas pelas empresas de um setor para a busca da vantagem competitiva) e o desempenho (das empresas e da economia de forma geral) (Barney; Hesterly, 2007). A partir das idéias de Bain, houve rápido crescimento de um ramo da economia que ficou conhecido como organização industrial e que procurava compreender porque alguns setores obtinham lucratividade superior a outros (Ghemawat, 2007). A organização industrial apresenta um enfoque determinístico visto que para sobreviver a organização precisa ser hábil para se adaptar às forças setoriais (Wright; Kroll; Parnell, 2000).

Devido ao foco do trabalho de Bain e seus sucessores em políticas públicas e a reduzida quantidade de variáveis estruturais utilizada para explicar a lucratividade do setor, a organização industrial não impactou rapidamente a estratégia de negócios; entretanto esses aspectos foram endereçados por Michael Porter que estudou a estrutura setorial e as estratégias de negócios (Ghemawat, 2007). O modelo das forças que dirigem a concorrência (Porter, 1980) representa uma ampliação da arena para a condução da análise competitiva, pois estudos anteriores sobre o ambiente competitivo somente focavam nas empresas que eram concorrentes diretos e não consideravam os outros atuais e potenciais concorrentes nem possíveis clientes e por quem eram atendidos (Hitt; Ireland; Hoskisson, 2008).

Para Porter (1980), a concorrência em um setor vai além do comportamento dos concorrentes e depende da estrutura do setor, que envolve um conjunto de forças (rivalidade entre as empresas existentes, ameaça de novos entrantes, ameaça de produtos ou serviços substitutos, poder de negociação dos fornecedores e poder de negociação dos compradores) que determina a lucratividade do setor com base no retorno de longo prazo sobre o investimento realizado. O modelo das cinco forças apresenta três implicações relevantes para a escolha e implementação de estratégias: (i) a descrição das fontes mais tradicionais de ameaças ambientais locais em dado setor, (ii) a utilidade para caracterização do nível geral de ameaça em um setor específico e (iii) a possibilidade de previsão do nível médio de desempenho das empresas que atuam num setor, segundo a lógica do modelo E-C-D (Barney; Hesterly, 2007). O modelo das cinco forças de Porter apresenta algumas limitações, dentre as quais se destacam: (i) reduzida atenção aos fatores que possuem potencial para afetar a demanda, (ii) foco no setor como um todo ao invés de empresas do referido setor, (iii) falta de consideração do papel do governo, com exceção das situações em que este atua como fornecedor ou comprador e (iv) abordagem qualitativa com utilidade para avaliar tendências porém sem poder quantificar probabilidades (Besanko et al, 2006).

Brandenburger e Nalebuff (1996) propuseram o modelo da coopetição em que a empresa compete e coopera simultaneamente com outras organizações por meio de interações que se configuram uma rede de valor composta por clientes, fornecedores, competidores e comple- 
mentadores, sendo os últimos uma parte que torna a oferta da empresa mais atrativa para os primeiros. Diferentemente de Porter, que entende que outras empresas (compradores, fornecedores ou concorrentes) podem representar ameaças à lucratividade, Brandenburger e Nalebuff arguem que essas interações podem ser tanto negativas quanto positivas; portanto a rede de valor proposta por Brandenburger e Nalebuff aborda pontos cegos da visão de Porter ao considerar as oportunidades além das ameaças à lucratividade (Besanko et al, 2006).

Os modelos das cinco forças de Porter e da rede de valor de Brandenburger e Nalebuff provêm estruturas que viabilizam uma análise sistemática de questões econômicas e complexas que facilitam tarefas como: (i) avaliação do desempenho setorial e empresarial, (ii) identificação dos principais fatores que afetam o desempenho nas relações comerciais verticais e nas horizontais concorrentes, (iii) determinação do impacto das mudanças ambientais no desempenho e (iv) identificação de oportunidades e ameaças no cenário de negócios (Besanko et al, 2006).

O modelo da hipercompetição aborda os resultados das manobras estratégicas de concorrentes globais e inovadores com base na velocidade e agressividade de interação nas arenas de preço e qualidade, capacidade de criação de know-how e estabelecimento de vantagens pioneiras, luta para criação e destruição de fortalezas e competição para o acúmulo de reservas financeiras e neutralização destas junto aos concorrentes (D’Aveni, 1995).

Ghemawat (2007) enfatizou a importância do mapeamento de cenários para a compreensão das variações que ocorrem no ambiente e sua incorporação às ações estratégicas da empresa como um processo mais estruturado do que a abordagem SWOT (forças, fraquezas, oportunidades e ameaças), visto que a análise de cenários vai além dos concor- rentes diretos e considera outros aspectos importantes como a análise de demandaoferta, as cinco forças que dirigem a concorrência (Porter, 1980) e a rede de valor (Brandenburger; Nalebuff, 1996).

McGahan (2004) propôs um modelo para entender como os setores evoluem segundo quatro alternativas de trajetórias (progressiva, criativa, intermediária ou radical) possibilitando identificar a situação atual do setor e como formular estratégias para cada uma das trajetórias e proporcionando benefícios como: (i) determinação a respeito de ameaças sobre as atividades centrais ou ativos do setor, (ii) identificação das implicações de mudanças estruturais antes do que os competidores, (iii) distinção entre desenvolvimentos importantes e distrações temporárias, (iv) avaliação das opções para competir em cada trajetória, (v) decisão a respeito das formas apropriadas de inovação, (vi) otimização dos trade-offs para cada situação e (vii) utilização dos pontos fortes atuais para conquista de vantagens competitivas.

Grant (2005) sugere uma estrutura para a análise setorial que contempla vários aspectos apontados por outros autores e que envolve as seguintes etapas: (i) descrição da estrutura do setor, (ii) previsão de lucratividade do setor e (iii) estratégias para alteração da estrutura do setor.

\subsection{Nanociência e Nanotecnologia (NN): Aspectos Essenciais e Importân- cia para a Sociedade}

Nos últimos anos, os avanços tecnológicos têm permitido o desenvolvimento de duas áreas da ciência que são multidisciplinares e abrangem suas atividades cruzando tecnologia da informação, ciências exatas, ciências biológicas e engenharias: a nanociência e a nanotecnologia. A nanociência é o estudo dos fenômenos e manipulação dos materiais em escala atômica, molecular e macromolecular, onde as pro- 
priedades destes materiais diferem significativamente de seus respectivos materiais em reticulado estendido. A nanotecnologia é o desenvolvimento, caracterização, produção e aplicação de estruturas, dispositivos e sistemas pelo controle da forma e tamanho dos materiais em nano escala (Melo; Pimenta, 2004). Ambas tratam do desenvolvimento e aplicações de nanoestruturas e nanodispositivos utilizando-se das propriedades físicas, químicas, elétricas e óticas de novos materiais. Estes envolvem materiais poliméricos, metálicos, cerâmicos e biológicos. Para Maciel et al. (2003), a Nanociência figura como uma das áreas estratégicas para o desenvolvimento tecnológico neste século. A nanotecnologia e nanociência vêm impulsionando à inovação e evolução do conhecimento, marcando presença em quase todas as revistas científicas da atualidade.

As nanoestruturas são, em princípio, os menores sistemas feitos pelo homem e apresentam novas propriedades físicas, químicas e biológicas. Segundo Pinto (2006), o ponto fundamental da nanotecnologia é aprender a explorar estas propriedades, manufaturálas e empregá-las eficientemente.

$\mathrm{O}$ estudo de materiais cujos tamanhos das partículas que os constituem encontram-se na faixa dos nanômetros, os chamados de nanomateriais, ganhou importância significativa no final do século XX, levando ao aparecimento e consolidação de toda uma área do conhecimento, hoje reconhecida como Nanociência e Nanotecnologia. De maneira direta, pode-se apoiar a nanotecnologia e nanociência sobre um grande alicerce: o fato de que as propriedades dos materiais, da maneira pela qual são conhecidas, são fortemente dependentes do tamanho das partículas deste material. Em outras palavras, todas as propriedades dos materiais (óticas, elétricas, magnéticas, de transporte, catalíticas, entre outras), da forma pela qual são conheci- das, manifestam-se a partir de um determinado tamanho, chamado de crítico. Quando as partículas deste material estão abaixo deste tamanho crítico, esta propriedade se torna diferenciada (Zarbin, 2007).

A ciência e tecnologia dos materiais nanoestruturados é uma área multidisciplinar e interdisciplinar de pesquisa e desenvolvimento que possui um potencial capaz de revolucionar os métodos nos quais materiais e produtos são criados e a faixa e natureza de funcionalidades que podem ser acessados. A essência da nanotecnologia é a habilidade de trabalhar em nível atômico, gerando estruturas maiores (nanoestruturas) com uma organização molecular mais definida (Couto, 2006). Nano (do grego "anão") é um prefixo usado nas ciências para designar uma parte em um bilhão e, assim, um nanômetro $(1 \mathrm{~nm})$ corresponde a um bilionésimo de um metro.

A Nanotecnologia pode ser considerada como um conjunto de ações de pesquisa, desenvolvimento e inovação que são obtidas graças às especiais propriedades da matéria organizada a partir de estruturas de dimensões nanométricas. A nanotecnologia vem despertando muito interesse nas comunidades científicas e ao longo das últimas décadas muitos esforços foram feitos no sentido de atingir o tão desejado controle em nível atômico e molecular sobre os processos industriais. Segundo Pina et al. (2006), pela reduzida escala em que atua esta tecnologia, é possível sintetizar a matéria da forma mais adequada à utilização desejada. Ressalta-se que atualmente a nanotecnologia é hoje um dos principais focos das atividades de pesquisa, desenvolvimento e inovação em todos os países industrializados.

Conforme discutido anteriormente, a nanotecnologia consiste numa revolução tecnológica de grande abrangência. Suas consequências trazem enormes avanços ao bem estar material 
das sociedade, principalmente pela maior eficácia da aplicabilidade devido às propriedades físicas e químicas dos materiais nanoestruturados. Entretanto, para tornar possível o surgimento de outras aplicações tecnológicas, ainda existem diversos aspectos científicos a serem explorados.
Segundo Munford (2002), o estudo de nanoestruturas é de extrema importância tanto do ponto de vista científico quanto tecnológico. De acordo com Roco (1999), Toma (2005) e ABDI (2010) algumas das possíveis aplicações da nanotecnologia estão presentes em diversas áreas, apresentadas no Quadro 1.

Quadro 1 - Síntese das possíveis aplicações da nanotecnologia.

\begin{tabular}{|c|c|}
\hline Setor & Exemplos de aplicações \\
\hline Indústria automobilística e aeronáutica & $\begin{array}{l}\text { Fabricação de materiais mais leves, pneus mais duráveis, } \\
\text { plásticos não inflamáveis e mais baratos. }\end{array}$ \\
\hline Indústria farmacêutica, biotecnológica e biomédica & $\begin{array}{l}\text { Criação de novos medicamentos baseados em nanoes- } \\
\text { truturas, de materiais para regeneração de ossos e teci- } \\
\text { dos, de sensores para glicose, } \mathrm{CO}_{2} \text { e colesterol. }\end{array}$ \\
\hline Indústria química e de materiais & $\begin{array}{l}\text { Produção de catalisadores mais eficientes, ferramentas } \\
\text { de corte mais duras, fluidos magnéticos inteligentes. }\end{array}$ \\
\hline Indústria eletrônica e de comunicações & $\begin{array}{l}\text { Armazenamento de dados, em telas planas, aumento da } \\
\text { velocidade de processamento. }\end{array}$ \\
\hline Setor energético & $\begin{array}{l}\text { Economia de energia ao utilizar materiais mais leves e } \\
\text { circuitos menores, fabricação de células fotovoltaicas e } \\
\text { fotoeletroquímicas, fabricação de dispositivos orgânicos } \\
\text { emissores de luz, aplicações de nanotubos de carbono em } \\
\text { células solares, fabricação de nanocatalisadores para } \\
\text { produção de hidrogênio, produção de células a com- } \\
\text { bustível, fabricação de nanomateriais para armazenagem } \\
\text { de hidrogênio. }\end{array}$ \\
\hline Setor de fabricação & $\begin{array}{l}\text { Desenvolvimento de novos microscópios e instrumentos } \\
\text { de medida, de ferramentas para manipular a matéria a } \\
\text { nível atômico, de bioestruturas, etc. }\end{array}$ \\
\hline Meio ambiente & $\begin{array}{l}\text { Desenvolvimento de membranas seletivas para remover } \\
\text { contaminantes ou sal da água, novas possibilidades de } \\
\text { reciclagem, fabricação de nanocatalisadores mais efi- } \\
\text { cientes e econômicos para conversão catalítica em esca- } \\
\text { pamentos automotivos, fabricação de nanossensores para } \\
\text { detecção de agentes tóxicos e vazamentos, fabricação de } \\
\text { nanodispositivos para separação de gases, desen- } \\
\text { volvimento de nanomembranas para purificação de água, } \\
\text { dessalinização e desintoxicação, de nanossensores para } \\
\text { detecção de contaminantes e agentes patogênicos, entre } \\
\text { outros. }\end{array}$ \\
\hline
\end{tabular}


Produtividade agrícola

Desenvolvimento de zeólitas nanoporosas para liberação lenta e controlada de água, de fertilizantes e agrodefensivos, de nanocápsulas liberadoras de herbicidas, de nanossensores para monitoração do solo e do desenvolvimento das plantas, de nanopartículas magnéticas para combater a contaminação do solo, produção de nanossensores para detecção de pragas, nanopartículas transportadoras ou liberadoras de pesticidas, inseticidas, ou de ação repelente.

\section{Metodologia}

A pesquisa realizada neste trabalho pode ser classificada quanto aos objetivos como exploratória. Segundo Cooper e Schindler (2008, p.128) "Os estudos exploratórios tendem a gerar estruturas soltas com o objetivo de descobrir futuras tarefas de pesquisa". O objetivo primário da exploração é desenvolver conceitos de forma mais clara, formular um problema de pesquisa melhor delineado ou desenvolver hipóteses para pesquisa adicional. Nestes termos, Gil (1999) destaca que a pesquisa exploratória é desenvolvida no sentido de proporcionar uma visão global acerca de determinado fato.

A seleção do setor brasileiro de nanotecnologia para ser analisado estrategicamente ocorreu com base em seu forte avanço tecnológico associado aos promissores benefícios que podem resultar de sua aplicação. Adicionalmente, ressalta-se a necessidade de avaliação dos desafios a serem superados para que as oportunidades possam ser capitalizadas.

Para Martins e Teophilo (2007), a Pesquisa Bibliográfica, estratégia de pesquisa utilizada neste estudo, é necessária para a condução de qualquer pesquisa científica. Uma pesquisa bibliográfica procura explicar e discutir um assunto, tema ou problema com base em referências publicadas em livros, periódicos, revistas, enciclopédias, dicionários, jornais, sites, CDs, anais de congressos etc. Dessa forma, busca conhecer, anali- sar e explicar contribuições sobre determinado assunto, tema ou problema. Neste contexto, o objetivo da interpretação de uma ideia ou conceito é levar o autor a dizer explicitamente o que está dito de forma implícita, mas que não deixaria de dizer explicitamente se alguém lhe perguntasse. Assim, segundo os autores, a ordem dos apontamentos de cada texto é cronológica: as informações são registradas à medida que a leitura avança. Um bom apontamento deve ser claro e preciso, não deixando dúvidas sobre seu significado.

Seguindo essa estratégia de Pesquisa, os dados foram coletados a partir de fontes secundárias que envolveram estudos setoriais, relatórios governamentais nacionais (Agência Brasileira de Desenvolvimento Industrial - ABDI) e estrangeiros (Icon Group International), além de trabalhos científicos publicados nas áreas de ciências sociais e tecnologia sobre o tema nanociência e nanotecnologia.

Para efetuar a organização e a análise dos dados utilizou-se neste estudo a estrutura de análise sugerida por Grant (2005) e Richardson (1999), utilizando-se das seguintes etapas definidas: (i) descrição da estrutura do setor, (ii) previsão de lucratividade do setor e (iii) estratégias para alteração da estrutura do setor.

\section{Análise e Discussão}

Inicia-se este tópico com uma visão geral sobre a nanotecnologia no 
Brasil e no mundo, posteriormente conduzimos a análise do setor brasileiro de nanotecnologia e conclui-se com a apresentação dos desafios e oportunidades desse setor no Brasil.

\subsection{A Nanotecnologia no Brasil e no Mundo}

A nanotecnologia está concentrando investimentos vultuosos no mundo. De acordo com Roco (2004), estima-se que a produção industrial anual excederá a um trilhão de dólares, entre 2010 e 2015, requerendo aproximadamente dois milhões de trabalhadores. Países como Estados Unidos (EUA), União Européia (EU) e Japão são os que apresentam o maior nível de desenvolvimento em nanotecnologia. Grande parcela do crescimento do mercado de nanotecnologia não provém da produção de nanomateriais básicos, mas, sim, da capacidade de alguns segmentos, como o farmacêutico e o de semicondutores, de transformar os nanomateriais básicos em produtos de alto valor agregado (ABDI, 2010).

Os EUA lideram o ranking pelo número de patentes em Nanotecnlogia. Nas posições seguintes, encontram-se Japão, China, Coréia e Alemanha. Segundo Canavez (2006), com base dos dados da ABDI (2010), as empresas que mais registraram pedidos de patentes foram: IBM, Hewlett-Packard, Mícron Tecnology, AMD, Texas Intruments, Intel e Motorola, Canon, AlcatelLucent, Seiko Epson, Sumitomo, Samsung, Mitsubish, Toshiba, Procter \& Gamble e a L'Oreal.

No ano de 2012, o mercado foi dominado por semicondutores, mas surgirão novas aplicações baseadas em nanotecnologias para os segmentos farmacêuticos e saúde, alimentos e outros bens de consumo, devido à estabilidade da cadeia de suprimento dos nanomateriais. Em 2015, prevê-se que $80 \%$ do mercado de US\$ 1,5 trilhão correspon- derão a aplicações de nanotecnologia para os segmentos farmacêuticos e saúde humana.

Segundo dados da ABDI (2010), o consumo global de todos os tipos de nanomateriais, em 2005, atingiu um volume de 9 milhões de toneladas métricas e US\$ 13,1 bilhões, com uma expectativa de que alcance 10,3 milhões de toneladas e US\$ 20,5 bilhões em 2010, considerando-se uma taxa de crescimento anual de 9,3\%, em valor. Materiais orgânicos não-poliméricos respondem pela maior parcela do consumo total, porém prevê-se que o consumo dos nanomateriais óxidos crescerá a uma taxa de $8,5 \%$ para $15,75 \%$, em 2010 . Já os materiais metálicos são o segundo maior segmento com mais de 21\% do mercado. Em 2010, o consumo dos materiais nanoparticulados decrescerá $54,6 \%$, enquanto que os consumos de filmes, monolíticos e compósitos deverão crescer a taxas de $25,0 \%, 17,4 \%$ e $3,0 \%$, respectivamente. Entre 2005 e 2011, prevê-se que intermediários como nanocompósitos de metal, de óxido metálico e cerâmicos aumentarão suas participações para $20 \%$ e $11,5 \%$, respectivamente, nesse período.

A comercialização é um ponto marcante na experiência nanotecnológica de países como os Estados Unidos, o Japão, a China, a Índia, os Tigres Asiáticos e os países da Europa. A política de se criar nichos de excelência na nanotecnologia é uma característica marcante da experiência desses países. O Brasil não conseguiu ainda criar um "triple-helix" efetivo, envolvendo um número maior de empresas do setor privado nacional, governo, e instituições acadêmicas. $\mathrm{O}$ país tem um modelo top-down, ao contrário de outros países ondea está no ênfase ao modelo "bottom-up" consultivo (GOUVEA, 2010).

\subsection{Análise do Setor Brasileiro de Nanotecnologia}


Para conduzir a análise do setor brasileiro de nanotecnologia, utilizamos a estrutura adaptada Grant (2005) que aborda um conjunto de etapas conforme apresentadas a seguir.

\subsubsection{Descrição da Estrutura do Setor}

O setor de nanotecnologia é considerado de elevada intensidade tecnológica e voltado ao desenvolvimento de tecnologias e suas aplicações em produtos para diversos setores da economia. No Brasil ainda não se verifica a atuação marcante de grandes empresas, contudo em âmbito global, várias se destacam.

Ressalta-se uma lista de empresas que integram o mercado global de nanomateriais e que se encontram relacionadas no estudo da BCC Research: Basf AG; Bayer AG; Biophan Technologies; Capacitive Deionization Technology Systems Inc.; Covion Organic Semiconductors $\mathrm{GmbH}$; Degussa AG; Dendritic Nanotechnologies Inc.; E. I. du Pont de Nemours and Company; Eastman Kodak Co.; Eka Chemicals Colloidal Silica Group; Nanophase Technologies Corp.; Rhodia S.A.; Nalco Chemical Co.; Nanogate Technologies GmbH; NanoGram Corp.; Ishihara Sangyo Kaisha Ltd.; UltraDots Inc.; e Zyvex Corp. (ABDI, 2010).

\subsubsection{Previsão de Lucratividade do Setor}

A previsão de lucratividade do setor envolveu a análise do ambiente externo e da atratividade do setor para compreender como essas influenciam sua estrutura.

\subsubsection{Análise do ambiente externo}

A análise do ambiente externo foi conduzida por meio da estrutura PESTEL avaliando as dimensões política, econômica, social, tecnológica, ambiental e legal.

a) Política: Segundo dados do relatório da ABDI (ABDI, 2010), desde o ano de 2000, o governo brasileiro vem empreendendo esforços para a definição de um programa para o desenvolvimento e a disseminação das nanociências e da nanotecnologia. $\mathrm{O}$ governo brasileiro entre 2000 e 2007, investiu por meio de suas universidades e centros de pesquisa R\$ 160 milhões na pesquisa da nanotecnologia. Somando os investimentos do setor privado, estimase um total de R\$ 320 milhões no período. Os recursos, ainda que modestos comparados com investimentos feitos em outros países e mesmo no Brasil nos últimos anos, permitiram que as algumas redes se consolidassem.

O Brasil vem investindo cada vez mais em nanotecnologia. Estudos relacionados à nanotecnologia no Brasil vêm sendo incentivados pelo Conselho Nacional de Desenvolvimento Científico e Tecnológico (CNPq) e Ministério de Ciência e Tecnologia (MCT), desde 2001, momento em que foram criadas 4 redes de pesquisa em Nanociência e Nanotecnologia, nas seguintes áreas: Materiais nanoestruturados, Interfaces e Nanotecnologia molecular, Nanobiotecnologia e Nanodispositivos semicondutores (SANT'ANNA, ALENCAR, FERREIRA, 2013).

No entanto, quando se trata do desenvolvimento de inovações, observase que o país precisa incrementar os incentivos, apesar do Governo Federal estar investindo em uma série de instrumentos que visam fortalecer $\mathrm{O}$ potencial inovador brasileiro em N\&N, como os editais do $\mathrm{CNPq}$, da Financiadora de Estudos e Projetos (FINEP) e das Fundações de Amparo as Pesquisas (FAP's) com foco no desenvolvimento tecnológico de produtos, processos ou serviços nesta área, bem como em estratégias 
regulamentadoras como a Lei de Inovação, Lei no 10.973 de 2004.

b) Econômica: Segundo os dados da Agência Brasileira de Desenvolvimento Industrial (ABDI, 2010) o mercado total de produtos que incorporam nanotecnologias (incluindo semicondutores e eletrônicos) atingiu US\$ 135 bilhões em 2007, devendo alcançar US\$ 693 bilhões até o final de 2012 e cerca de US\$2,95 trilhões em 2015. Ressalta-se que os valores estimados de mercado não se referem apenas às nanotecnologias incorporadas aos produtos finais, mas sim aos valores dos produtos como um todo. O setor químico é o que ocupa atualmente a maior parcela do mercado global de nanotecnologia, seguido pelos semicondutores. Setores como farmacêutico, automotivo e defesa representam pequenas parcelas desse mercado.

c) Social: Mesmo que existam possibilidades de aplicação da nanotecnologia a diversas áreas de negócios sua compreensão pela sociedade ainda requer maiores esforços e esclarecimentos, pois não há transparência suficiente quanto aos potenciais benefícios e riscos relacionados à aplicação da nanotecnologia, especialmente com respeito aos setores de alimentação, saúde e cosméticos.

d) Tecnológica: As pesquisas de prospecção tecnológica que vem sendo desenvolvidas no Brasil indicam que as oportunidades de negócio em nanotecnologia tendem a surgir primeiramente nos mercados de cosméticos, produtos provenientes da indústria química (catalisadores, tintas, revestimentos) e petroquímica, plásticos, borrachas e ligas metálicas. O Brasil possui uma posição pouco expressiva em relação ao seu portfolio de patentes em nanotecnologia, principalmente quando comparado a países como Estados Unidos, China, Taiwan, Coréia e Índia. e) Ambiental: A avaliação dos riscos é uma questão-chave para o desenvolvimento comercial da nanotecnologia. A nanotecnologia oferece a perspectiva de grandes avanços que permitam melhorar a qualidade de vida e ajudar a preservar o meio ambiente. Entretanto, é bastante incipiente a discussão sobre os aspectos negativos da nanotecnologia no meio ambiente, pois como qualquer área da tecnologia que faz uso intensivo de novos materiais e substâncias químicas, ela traz consigo alguns riscos ao meio ambiente e à saúde humana.

Segundo Quina (2004), as mesmas características que tornam as nanopartículas interessantes do ponto de vista de aplicação tecnológica, podem ser indesejáveis quando essas são liberadas ao meio ambiente. O pequeno tamanho das nanopartículas facilita sua difusão e transporte na atmosfera, em águas e em solos, ao passo que dificulta sua remoção por técnicas usuais de filtração. Pode facilitar também a entrada e o acúmulo de nanopartículas em células vivas. De modo geral, sabe-se muito pouco ou nada sobre a biodisponibilidade, biodegradabilidade e toxicidade de novos nanomateriais.

Dentre os vários pontos importantes, ressalta-se a avaliação da toxidade dos nanotubos. As nanopartículas podem conter um potencial tóxico que a afetaria a saúde humana como comprova estudos em ratos que foram expostos a nanotubos de carbono. As vias respiratórias do ser humano é a principal rota de entrada das nanopartículas no organismo (Marques, 2008).

f) Legal: A análise dos aspectos relacionados a nanotecnologia fazem surgir implicações na esfera jurídica e debates no âmbito da sociedade, como a discussão realizada no parlamento norte americano, sob o título de Ato Nacional de Iniciativas de Emendas sobre Nanotecnologias de 2008 (National Nanotechology Initiative Amendments 
Act of 2008). Na ocasião surgiram definições sobre o tema, disponíveis no Ato de Pesquisa e Desenvolvimento da Nanotecnologia do Século 21 (21st Century Nanotechnology Research and Development Act), que entre os pontos abordados, apontou para a avaliação das consequências sociais e ambientais do uso dos nanomateriais, dispositivos e sistemas. No entanto, tais movimentos ainda não são suficientes para atender todos os pontos relacionados a regulamentação dessa tecnologia emergente. Há uma incipiente normatização no Brasil, por meio de portarias, no âmbito do Ministério da Ciência e Tecnologia (MCT), com objetivos de criar a administração e o sistema nacional de nanotecnologia, assim como prover interação com outros países no tema. Nada se aborda sobre segurança. Os objetivos são principalmente os de divulgar as realizações brasileiras no campo, parceria com outros países e pouco se aborda em aspectos de meio ambiente $\mathrm{e}$ impactos sociais desta nova descoberta. A ausência de cautela em relação à nanotecnologia ainda é marcante, contudo, o Direito, em seus vários ramos, apresenta ferramentas para proteger o bem ambiental.

\subsubsection{Análise da atratividade do setor}

A análise de atratividade do setor foi conduzida a partir dos modelos das cinco forças (Porter, 1980), da hipercompetição (D’Aveni, 1995), da coopetição (Brandenburger \& Nalebuff, 1996) e das trajetórias das indústrias (McGahan, 2004) e está apresentada a seguir por meio dos tópicos, dinâmica competitiva, entrantes e substitutos, fornecedores e clientes e trajetória de evolução:

a) Dinâmica competitiva: $\mathrm{O}$ setor de nanotecnologia ainda se encontra em crescimento e, dessa forma, não existe concentração relevante de participantes que eleve significativamente a rivalidade. Nota-se a existência de certa diversidade de empresas devido à amplitude de soluções que essas desenvolvem para vários setores e segmentos do mercado brasileiro. Essa condição proporciona a diferenciação de produtos em razão das particularidades nos nanocomponentes que são utilizados nas diferentes aplicações. Demonstrando consistência com o estudo de D'Aveni (1995), a competição encontra-se em um nível moderado no qual se evita a competição direta devido a segmentação dos mercados e se verifica um reduzido número de protagonistas que auferem lucros sustentáveis.

b) Entrantes, substitutos e complementadores: A entrada no setor requer a superação de algumas barreiras, dentre as quais se destacam o capital para investimento, o acesso ao conhecimento científico e adequação às legislações. A necessidade de investimento é um ponto determinante para atuação nesse setor devido ao elevado custo para exploração tecnológica. O conhecimento científico refere-se à condução de pesquisa básica para identificação de novas propriedades dos nanomateriais e seus potenciais usos. O governo exerce um papel regulador que ainda não está claro quanto à atuação de empresas de nanotecnologia, sendo sua postura predominantemente restritiva e dificultadora. Os produtos que são baseados em nanotenologia e que usam nanocomponentes podem atuar como substitutos de produtos que atualmente dominam o mercado, contudo devido ao estado de evolução desse setor ainda não se nota claramente a existência de substitutos para os nanoprodutos. Além dos substitutos, se destacam os complementadores os quais, consistentemente com Brandenburger e Nalebuff (1996), viabilizam a formação 
de redes de valor e elevam a atratividade dos produtos e serviços.

\section{c) Fornecedores e clientes:} Devido ao conhecimento das propriedades dos nanomateriais e suas aplicações, os fornecedores demonstram elevado poder de barganha, contudo esses necessitam ser capazes de comprovar os benefícios que podem ser proporcionados pelas suas soluções. Os compradores normalmente são empresas que buscam soluções para aprimorar o desempenho de seus produtos por parte dos usuários finais, os quais ainda se mostram reticentes à aceitação da nanotecnologia devido às incertezas relativas à sua aplicação.

d) Trajetória de evolução: $\mathrm{O}$ setor de nanotecnologia apresenta uma trajetória radical de evolução, pois conforme sugerido por McGahan (2004) utiliza novas abordagens para criação de valor que tornam obsoletos os principais ativos e atividades da empresa. Essa obsolescência pode resultar das novas tecnologias nano e do emprego dos nanomateriais, sendo que sua ocorrência se dá em fases consistentes com a proposta de McGahan (2004), que envolvem o surgimento de pequenos segmentos (emergência), o aumento da eficiência das operações (convergência), a utilização das abordagens antigas e novas (coexistência) e a criação de valor a partir das novas abordagens (dominação).

Com base na análise do ambiente externo e do setor de nanotecnologia, constatamos que a previsão de lucratividade desse setor depende primeiramente das tendências das forças externas que podem influenciar fortemente a atratividade setor. Nas atuais circunstâncias entendemos que a atratividade do setor de nanotecnologia ainda é incipiente com a atuação de algumas organizações pioneiras mas com muitas observando cautelosamente os rumos do setor antes de realizar seus comprometimentos estratégicos.

\subsubsection{Estratégias para alteração da estrutura do setor}

O Brasil não possui ainda uma base de dados sistematizada sobre o mercado dos produtos, processos e serviços baseados em nanotecnologia. É oportuno conceber tal sistemática considerando a cadeia de valor da nanotecnologia e seus componentes, alternativamente aos métodos convencionais de estudos de mercado. Estudos de prospecção tecnológica relacionados com a gestão estratégica poderão dar os primeiros passos rumo ao futuro, considerando-se que o país precisará escolher assertivamente os nichos em nanotecnologias nos quais deverá atuar e, simultaneamente, "catalisar" as relações entre pesquisas e o setor produtivo com a previsão das futuras margens operacionais.

A importância da divulgação de novas tecnologias por meio de artigos científicos tem contribuído para o aumento dos investimentos em Nanotecnologia. Segundo dados da ABDI (2010), o número de trabalhos em nanociência cresceu a uma taxa anual de $16 \%$, durante o período 1996-2006, medida pelo número total de publicações indexadas na base Scopus no mesmo período. Considerando esse total de publicações, o EUA vêm liderando, com 102.000 trabalhos, respondendo por $27 \%$ da produção mundial. O Brasil ocupa a $25^{\circ}$ posição no ranking geral de publicações, com 4.358 trabalhos publicados.

Nos últimos anos o Brasil tem avançado consideravelmente no desenvolvimento de ações em Ciência, Tecnologia e Inovação (C\&T\&I), com resultados concretos na produção científica, tecnológica e formação de recursos humanos em áreas consideradas estratégicas, particularmente em determinados campos de nanotecnologia e nanociência $(\mathrm{N} \& \mathrm{~N})$. No entanto, a oferta de produtos, processos e serviços 
ligados à nanotecnologia no Brasil não vem acompanhando os indicadores da produção científica, no qual o país se encontra hoje na $25^{\mathrm{a}}$ posição do ranking mundial, de acordo com os critérios adotados no estudo da Science-Metrix (2008). Considerando-se todos os componentes da cadeia de valor, constata -se um reduzido número de empresas que incorporam nanotecnologias em seus produtos ou processos ou que fabricam nanomateriais, nanointermediários ou nanoferramentas.

Segundo Toma (2005), no caso especial da Nanotecnologia, o desenvolvimento é uma questão ainda mais complexa, pois a abordagem inter e multidisciplinar também deve ser estendida às empresas, e não apenas para o setor acadêmico. A esse aspecto, deve ser adicionado o fator econômico e a falta de recursos humanos para desenvolver a Nanotecnologia. O fator econômico é especialmente preocupante, pois laboratórios de qualidade em Nanotecnologia exigem dispêndios de milhões de dólares em equipamentos sofisticados, instalações e insumos. Esse ponto aponta para a necessidade de novos modelos de organização de pesquisa, a fim de evitar que a alta tecnologia acabe se concentrando em empresas e instituições de maior porte, levando à exclusão das pequenas empresas, justamente onde o potencial de inovação tende a ser mais acentuado e precisa ser estimulado. Para isso, deveriam ser identificados os laboratórios estratégicos nas redes que, com o devido suporte, poderiam estender o uso das facilidades conseguidas para os demais usuários, evitando sua concentração em laboratórios específicos, isolados.

\section{Considerações Finais}

Este trabalho foi conduzido com o objetivo de analisar o setor brasileiro de nanotecnologia para identificar suas oportunidades e desafios. A partir de uma pesquisa documental junto a várias fontes associadas à análise foi constatado que esse setor ainda encontra-se muito incipiente no Brasil, necessitando de suporte nas esferas pública e privada não somente para o desenvolvimento tecnológico mas também para a comercialização dos produtos e serviços resultantes da aplicação da nanotecnologia.

As principais oportunidades identificadas incluem, mas não se limitam (i) à aplicação da nanotecnologia ao desenvolvimento e aprimoramento de produtos em diversos setores como agroindústria, alimentos, cosméticos, entre outros e (ii) ao desenvolvimento científico na área de ciência e tecnologia e áreas correlatas.

Portanto, os principais desafios para a evolução do setor de nanotecnologia no Brasil envolvem a superação de obstáculos relacionados (i) ao estabelecimento de marcos regulatórios que definam claramente as possibilidades de sua aplicação e as responsabilidades sobre os riscos assumidos, (ii) à definição de políticas de investimento que viabilizem o avanço científico e a difusão tecnológica da nanotecnologia e (iii) à criação de mecanismos de governança que facilitem a comunicação com a sociedade e proporcionem maior transparência sobre os benefícios e riscos decorrentes da aplicação da nanotecnologia.

Em relação à complexidade envolvida na regulação das nanotecnologias, um dos maiores desafios que os governos mundiais já estão enfrentando é explorar adequadamente uma série de quadros regulamentares flexíveis, específicos para as nanotecnologias. Nesses quadros, vários graus de regulação dependerão de cada fase do desenvolvimento defendidas, por Bowman e Hodge (2007), implicando em demandas organizacionais referentes à capacidade 
do sistema regulatório de se adaptar de forma flexível e equilibrada aos benefícios e riscos decorrentes dos avanços das nanotecnologias. Segundo dados do relatório do projeto ObservatoryNano, publicado em junho de 2010, os principais aspectos técnicos e desafios associados à criação de regimes regulatórios para a nanotecnologia são: (a) diversidade de materiais e aplicações; (b) falta de conhecimento sobre os nanomateriais; (c) falta de padronização na nomenclatura, métricas e materiais; e (d) propriedade da informação (ObservatoryNano, 2010).

Acredita-se que o presente estudo traz contribuições tanto acadêmicas quanto práticas. Academicamente, apresenta a nanotecnologia e suas aplicações não somente do ponto de vista tecnológico mas também mercadológico. As contribuições práticas relacionam-se com a compreensão da evolução desse setor e da possibilidade de melhor interpretar suas possíveis trajetórias de desenvolvimento.

Apesar dos esforços empenhados na elaboração deste trabalho, algumas limitações devem ser consideradas. A pesquisa documental foi utilizada como uma abordagem inicial do tema e pode ser aprofundada com uma pesquisa de campo junto às organizações e órgãos que possuem envolvimento e interesse no setor de nanotecnologia.

Como grande parte da literatura sobre nanotecnologia ainda é predominantemente estrangeira e sobre mercados externos ao Brasil, existem grandes possibilidades de estudos nessa área para avaliar aspectos específicos desse setor como estratégias das empresas, empreendedorismo e criação de novos negócios, cadeias e redes de valor, políticas tecnológicas e de suporte ao desenvolvimento setorial, entre outros.

\section{Referências}

ABDI - Agência Brasileira de Desenvolvimento Industrial. Panorama nanotecnologia. Centro de Gestão e Estudos Estratégicos. Brasília, 180p, 2010.

ALTENSTETTER, C. Medical device regulation and nanotechnologies: determining the role of patient safety concerns in policymaking. Law \& Policy, v. 33, n. 2, p. 227-255, 2011.

BAIN, J. S. Industrial organization. New York: John Wiley \& Sons, 1968.

BARNEY, J. B.; HESTERLY, W. S. Administração estratégica e vantagem competitiva. São Paulo: Pearson Prentice Hall, 2007.

BATISTA, A. J. S.; PEPE, V. L. E. Os desafios da nanotecnologia para a vigilância sanitária de medicamentos. Ciência \& Saúde Coletiva, v. 19, n.7, p. 2105-2114, 2014.

BESANKO, D.; DRANOVE, D.; SHANLEY, M.; SCHAEFER, S. A economia da estratégia. 3. ed. Porto Alegre: Bookman, 2006.

BOWMAN, D. M.; HODGE, G. A. Nanotechnology 'Down Under': Getting on top of Regulatory Matters. Nanotechnology Law and Business Journal, v. 4, n. 2, p. 223-233, 2007.

BRANDENBURGER, A. M.; NALEBUFF, B. J. Co-opetition. New York: Currency Doubleday, 1996.

BUZBY, J. C. Nanotechnology for food applications: more questions than answers. The Journal of Consumer Affairs, v. 44, n. 3, p. 528-545, 2010.

CANAVEZ, M. J. M. O uso da nanotecnologia nas empresas: um estudo de caso no setor de cosméticos. 
Dissertação de mestrado, Universidade Federal do Paraná, Curitiba, PR, Brasil, 2011.

CARPENTER, M. A.; SANDERS, W. G. Strategic management: a dynamic perspective. Upper Saddle River, NJ: Pearson Prentice Hall, 2007.

CERTO, S. C.; PETER, J. P.; MARCONDES, R. C.; CESAR, A. M. R. Administração estratégica: planejamento e implantação de estratégias. São Paulo: Pearson, 2010.

CONTI, J.; SATTERFIELD, T.; HARTHORN, B. H. Vulnerability and social justice as factors in emergent U.S. nanotechnology risk perceptions. Risk Analysis, v. 31, n. 11, p. 1734-1748, 2011.

COOPER, D.; SCHINDLER, P. Métodos de Pesquisa em Administração. 7. ed. Porto Alegre: Bookman, 2008.

D'AVENI, R. A. Hipercompetição: estratégias para dominar a dinâmica do mercado. Rio de Janeiro: Campus, 1995.

DESS, G. G.; LUMPKIN, G. T.; EISNER, A. B. Strategic management: creating competitive advantages. 3rd. ed. New York, NY: McGraw-Hill Irvin, 2007.

DOERING, R. Potential opportunities for nanotechnology in electronics manufacturing. Solid State Technology, 1215, 2011.

DOUBLEDAY, R. Risk, public engagement and reflexivity: alternative framings of the public dimensions of nanotechnology. Health, Risk \& Society, v. 9, n. 2, p. 211-227, 2007.

ERDOGAN, Y. Nanotechnology in cement \& concrete industry. Cement \&
Concrete World, v. 16, n. 94, p. 61-75, 2011.

FALKNER, R.; JASPERS, N. Regulating nanotechnologies: risk, uncertainty and the global governance gap. Global Environmental Politics, v. 12, n. 1, p. 30-55, 2012.

FEATHERSTONE, D. J. Nanotechnology Patents: a snapshot of nanotechnology patenting Through an Analysis of 10 Top Nanotech Patents. Intellectual Property \& Technology Law Journal, v. 16, n. 12, p. 19-24, 2004.

FERNÁNDEZ-RIBAS, A. International patent strategies of small and large firms: an empirical study of nanotechnology. Review of Policy Research, v. 27, n. 4, p. 457-473, 2010.

FESTEL, G.; KRATZER, J. The founding angels investment model - case studies from the field of nanotechnology. Journal of Business Chemistry, v. 9, $\mathrm{n}$. 1, p. 19-29, 2012.

FINK, M.; HARMS, R.; HATAK, I. Nanotechnology and ethics: the role of regulation versus self-commitment in shaping researchers' behavior. Journal of Business Ethics, v. 109, n. 4, p. 569581, 2012.

FOLADORI G.; FIGUEROA S.; EDGARD Z.; INVERNIZZ I. N. Características distintivas del desarrollo de las nanotecnologias en américa latina. Sociologias, v. 14, n.30, p. 330-363, 2012.

FRY, J. N.; KILLING, J. P. Strategic analysis and action. 4th. ed. Scarborough, Ontario: Prentice Hall Canada, 2000 .

Future prospects for nanotechnology in food packaging. Food Engineering \& Ingredients, v. 36, n. 4, p. 36-39, 2011. 
GHAZINOORY, S.; GHAZINOURI, R. Nanotechnology and sociopolitical modernity in developing countries: case study of Iran. Technological and Economic Development of Economy, v. 15, n. 3, p. 395-417, 2009.

GHAZINOORY, S.; SOOFI, A. Modifying BSC for national nanotechnology development: an implication for "social capital" role in NIS theory. Technological and Economic Development of Economy, v. 18, n. 3, p. 487-503, 2012.

GHEMAWAT, P. A estratégia e o cenário de negócios. 2. ed. Porto Alegre: Bookman, 2007.

GIL, A. Métodos e técnicas de pesquisa social. 5. ed. São Paulo: Atlas, 1999.

GOUVEA, R. Nanotecnologia, Um Novo Paradigma de Desenvolvimento Econômico: Uma Análise da Experiência Internacional \& Brasileira. Estratégia, v. 9, n. $8,2010$.

GRANT, R. M. Contemporary strategy analysis. 5th. ed. Oxford, UK: Blackwell Publishing, 2005.

GROVES, C.; FRATER, L.; LEE, R.; STOKES, E. Is there room at the bottom for CSR? corporate social responsibility and nanotechnology in the UK. Journal of Business Ethics, v. 101, n. 4, p. 525$552,2011$.

HANDY, R. D.; SHAW, B. J. Toxic effects of nanoparticles and nanomaterials: implications for public health, risk assessment and the public perception of nanotechnology. Health, Risk \& Society, v. 9, n. 2, p. 125-144, 2007.

IGAMI, M.; OKAZAKI, T. Capturing nanotechnology's current state of development via analysis of patents [Working Paper $N^{0}$ 2007/4]. Statistical Analysis of Science, Technology and Industry -
Organisation for Economic Cooperation and Development. Paris, 2007.

JOHNSON, G.; SCHOLES, K.; WHITTINGTON, R. Explorando a estratégia corporativa: textos e casos. Porto Alegre: Bookman, 2007.

KHADZHIEV, S. N. Nanoheterogeneous catalysis: a new sector of nanotechnologies in chemistry and petroleum chemistry (a review). Petroleum Chemistry, v. 51, n. 1, p. 1-15, 2011.

KUZMA, J.; PRIEST, S. Nanotechnology, risk, and oversight: learning lessons from related emerging technologies. Risk Analysis, v. 30, n. 11, p. 16881698, 2010.

KUZMA， J.; ROMANCHEK, J.; KOKOTOVICH, A. Upstream oversight assessment for agrifood nanotechnology: a case studies approach. Risk Analysis, v. 28, n. 4, p. 1081-1098, 2008.

Lei ${ }^{0} 10.973$, de 02/12/2004, que dispõe sobre incentivos à inovação e à pesquisa científica e tecnológica no ambiente produtivo, e dá outras providências; http:// www.planalto.gov.br/ccivil_03/_ato2004 -2006/2004/lei/110.973.htm.

LINDQUIST, E.; MOSHER-HOWE, K. N.; LIU, X. Nanotechnology...what is it good for? (absolutely everything): a problem definition approach. Review of Policy Research, v. 27, n. 3, p. 255-271, 2010.

LINTON, J. D.; WALSH, S. T. Introduction to the field of nanotechnology ethics and policy. Journal of Business Ethics, v. 109, n. 4, p. 547-549, 2012.

LIU, X.; KAZA, S.; ZHANG, P.; CHEN, H. Determining inventor status and its effect on knowledge diffusion: a study on nanotechnology literature from China, Russia, and India. Journal of the 
American Society for Information Science and Technology, v. 62, n. 6, p. 1166-1176, 2011.

LU, L. Y. Y.; LIN, B. J. Y.; LIU, J. S.; YU, C-Y. Ethics in Nanotechnology: What's Being Done? What's Missing? Journal of Business Ethics, v. 109, n. 4, p. 583-598, 2012.

MARTINS, G. A, THEÓPHILO, C. $\mathrm{R}$, Metodologia da Investigação Científica para Ciências Sociais Aplicadas. São Paulo, Atlas, 2007.

MCGAHAN, A. How industries evolve: principles for achieving and sustaining superior performance. Boston, MA: Harvard Business School Press, 2004.

MEYER, M.; LIBAERS, D.; PARK, J$H$. The Emergence of Novel Sciencerelated Fields: regional or technological patterns? Exploration and Exploitation in United Kingdom Nanotechnology. Regional Studies, v. 45, n. 7, p. 935-959, 2011.

MOINDDIN, Q. M.; RASHDI, P. I. S.; BHUTTO, A. Innovations in nanotechnology organizations. International Journal of Innovation and Technology Management, v. 7, n. 4, p. 377-388, 2010.

MUNARI, F.; TOSCHI, L. Do venture capitalists have a bias against investment in academic spin-offs? Evidence from the micro- and nanotechnology sector in the UK. Industrial and Corporate Change, v. 20, n. 2, p. 397-432, 2010.

NERLICH, B.; CLARKE, D. D.; ULPH, F. Risks and benefits of nanotechnology: How young adults perceive possible advances in nanomedicine compared with conventional treatments. Health, Risk \& Society, v. 9, n. 2, p. 159-171, 2007.
ObservatoryNano. ICT Sector Focus Report. Printed Electronics, 2010.

OTT, I.; PAPILLOUD, C. Convergence or mediation? Experts of vulnerability and the vulnerability of experts' discourses on nanotechnologies: a case study. The European Journal of Social Science Research, v. 21, n. 1, p. 41-64, 2008.

PIDGEON, N.; ROGERS-HAYDEN, T. Opening up nanotechnology dialogue with the publics: risk communication or 'upstream engagement'? Health, Risk \& Society, v. 9, n. 2, p. 191-210, 2007.

PIDGEON, N.; HARTHORN, B.; SATTERFIELD, T. Nanotechnology risk perceptions and communication: emerging technologies, emerging challenges. Risk Analysis, v. 31, n. 11, p. 1694-1700, 2011.PYRRHO, M.; SCHRAMM, F. R. A moralidade da nanotecnologia. Cad. Saúde Pública, v. 28, n. 11, p. 2023-2033, 2012.

PORTER, M. E. Competitive Strategy. New York: The Free Press, 1980.

PRESCHITSCHEK, N.; BRESSER, D. Nanotechnology patenting in China and Germany - a comparison of patent landscapes by bibliographic analyses. Journal of Business Chemistry, v. 7, n. 1, p. 3-13, 2010.

PRIEST, S.; LANE, T.; GREENHALGH, T.; HAND, L. J.; KRAMER, $\mathrm{V}$. Envisioning emerging nanotechnologies: a three-year panel study of South Carolina citizens. Risk Analysis, v. 31, n. 11, p. 1718-1733, 2011.

RASMUSSEN, B. Is the commercialisation of nanotechnology different? a case study approach. Innovation: Management, Policy \& Practice, v. 9, n. 1, p. 62-78, 2007. 
MEYER, M.; LIBAERS, D.; PARK, JH. Regional or Technological Patterns? Exploration and Exploitation in United Kingdom Nanotechnology. Regional Studies, v. 45, n. 7, p. 935-959.

REISCH, L. A.; SCHOLL, G.; BIETZ, S. 'Better safe than sorry': consumer perceptions of and deliberations on nanotechnologies. International Journal of Consumer Studies, v. 35, n. 6, p. 644-654, 2011.

RICHARDSON, R. J. Pesquisa social: métodos e técnicas. São Paulo: Atlas, 1999.

ROCHMAN, N. T.; Gumbira-Sa'id, E.; Daryanto, A.; \& Nuryartono, N. Analysis of Indonesian agroindustry competitiveness in nanotechnology development perspective using SWOT-AHP method. International Journal of Business and Management, v. 6, n. 8, p. 235-244, 2011.

ROCO, M. C. The US national nanotechnology initiative after 3 years (2001-2003). Journal of Nanoparticule Research, v. 6, n. 1, p. 1-10, 2004.

ROCO, M. C.; WILLIANS, S.; ALIVISATOS, P. Nanotechnology research directions. IWGN workshop report: vision for nanotechnology research and development in the next decade. WTEC, Loyola College: Maryland, 1999.

SÁ, C. M.; GEIGER, R. L.; HALLACHER, P. M. Universities and state policy formation: rationalizing a nanotechnology strategy in Pennsylvania. Review of Policy Research, v. 25, n. 1, p. 3-19, 2008.

SANT'ANNA, L. S, ALENCAR, M. S., FERREIRA, A. P. Patenteamento em nanotecnologia no Brasil: desenvolvimento, potencialidades e reflexões para o meio ambiente e a saúde humana. Química Nova, v. 36 n. 2, 2013.

SANTOS, F. F. A encruzilhada da nanotecnologia: inovação, tecnologia e riscos. RAC, v. 14, n. 5, p. 983-985, 2010.

SCHELLEKENS, M. Patenting nanotechnology in Europe: making a good start? an analysis of issues in law and regulation. The Journal of World Intellectual Property, v. 13, n. 1, p. 4776, 2010.

SCIENCE-METRIX. Nanotechnology World R\&D Report 2008. Serie R\&D Reports Examining Science and Technology. Montreal: Science-Metrix Inc., 185 p, 2008.

SHATKIN, J. A.; NORTH, W. Perspectives on risks of nanomaterials and nanotechnologies: advancing the science. Risk Analysis, v. 30, n. 11, p. 16271633, 2010.

SOZER, N.; KOKINI, J. L. Applications of nanotechnology in the food industry. Food Engineering \& Ingredients, v. 35 , n. 1, p. 12-15, 2010.

STAMPFLI, N.; SIEGRIST, M.; KASTENHOLZ, H. Acceptance of nanotechnology in food and food packaging: a path model analysis. Journal of Risk Research, v. 13, n. 3, p. 335-347, 2010.

STINNETT, R. Nanotechnology policy and education. Journal of Business Ethics, v. 109, n. 4, p. 551-552, 2012.

SYLVESTER, D. J.; ABBOTT, K. W.; MARCHANT, G. E. Regulation \& Governance, v. 3, n. 2, p. 165-185, 2009.

THOMAS, T. C.; ACUÑA-NARVAES, $R$. The convergence of biotechnology and nanotechnology: Why here, why now? Journal of Commercial Biotech- 
nology, v. 12, n. 2, p. 105-110, 2006.

THRONE-HOLST, H.; STRANDBAKKEN, P. "Nobody told me I was a nanoconsumer:" how nanotechnologies might challenge the notion of consumer rights. Journal of Consumer Policy, v. 32, n. 4, p. 393-402, 2009.

TOMA, H. E. Interfaces e organização da pesquisa no Brasil: da química à nanotecnologia. Quím. Nova, v. 28, S48-S51, 2005.

TSAI, C. The Nanotechnology Revolution: Ushering in a new wave of Toxic Torts? Intellectual Property \& Technology Law Journal, v. 24, n. 3, p. 2024, 2012.

URANGA, M. G.; KEREXETA, G. E. ; CAMPÀS-VELASCO, J. The dynamics of commercialization of scientific knowledge in biotechnology and nanotechnology. European Planning Studies, v. 15, n. 9, p. 1199-1214, 2007.

VAN BROEKHUIZEN, P.; REIJNDERS, L. Building blocks for a precautionary approach to the use of nanomaterials: positions taken by trade unions and environmental NGOs in the European nanotechnologies debate. Risk Analysis, v. 31 , n. 10, p. 1646-1657, 2011.

VAN LENTE, M. A. Building the new world of nanotechnology. Case Western Reserve Journal of International Law, v. 38, n. 1, p. 173-215, 2006.

WANG, J.; SHAPIRA, P. Partnering with universities: a good choice for nanotechnology start-up firms? Small Business Economics, v. 38, n. 2, p. 197215, 2012.

WILLIAMS, R. A.; KULINOWSKI, K. M.; WHITE, R.; LOUIS, G. Risk characterization for nanotechnology. Risk
Analysis, v. 30, n. 11, p. 1671-1679, 2010.

WILSON, W. A bit of gold and nanotechnology: bring viruses to light. Agriculture Research, April, 1-5, 2012.

WOOLLEY, J. L.; ROTTNER, R. M. Innovation policy and nanotechnology entrepreneurship. Entrepreneurship Theory and Practice, v. 32, n. 5, p. 791811, 2008.

WRIGHT, P.; KROLL, M. J.; PARNELL, J. Administração estratégica: conceitos. São Paulo: Atlas, 2000.

XENOS, M. A.; BECKER, A. B.; ANDERSON, A. A.; BROSSARD, D.; SCHEUFELE, D. A. Stimulating upstream engagement: an experimental study of nanotechnology information seeking. Social Science Quarterly, v. 92, n. 5, p. 1191-1214, 2011.

YAWSON, R. M.; KUZMA, J. Systems mapping of consumer acceptance of agrifood nanotechnology. Journal of Consumer Policy, v. 33, n. 4, p. 299322, 2010.

ZARBIN, A. J. Química de (nano) materiais. Quim. Nova, v. 30, n. 6, p. 14691479, 2007.

ZAWISLAK, P. A.; MARQUES, L. F.; ESTEVES, P.; RUBLESCKi, F. Technological trajectories and multidimensional impacts: further remarks on the nanotechnology industry. Journal of Business Chemistry, v. 7, n. 1, p. 47-64, 2010. 